\title{
Precoding Designs for Full-Duplex Multi-User MIMO Cognitive Networks with Imperfect CSI
}

\author{
Xuan-Xinh Nguyen, Ha Hoang Kha \\ Ho Chi Minh City University of Technology, VNU-HCM, Vietnam \\ Correspondence: Ha Hoang Kha, hhkha@hcmut.edu.vn \\ Communication: received 29 December 2018, revised 14 April 2019, accepted 15 April 2019 \\ Online publication: 30 June 2019, Digital Object Identifier: 10.21553/rev-jec.229 \\ The associate editor coordinating the review of this article and recommending it for publication was Dr. Huan Xuan Nguyen.
}

\begin{abstract}
This paper studies a cognitive radio (CR) network which consists of a full-duplex (FD) multi-user (MU) multipleinput multiple-output (MIMO) secondary user (SU) networks operating within the coverage of multiple primary users (PUs). It is assumed that the channel state information (CSI) matrices associated with SU systems are perfectly known whereas the CSI ones from SUs to PUs are imperfectly estimated. The problem of interest is to design robust precoding matrices at the SUs to maximize the CR sum rate subject to the SU transmit power constraints and harmful interference restrictions at PUs. Due to non-concavity of the objective function and intractability of robust PU interference constraints, the design problem is non-convex and challenging to directly solve. We exploit the difference of two concave functions to recast the sum rate objective function as a lower bounded concave one. In addition, a linear matrix inequality (LMI) transformation is used to handle the semi-infinite robust interference constraints. Then, the sequential convex programming method is carried out to iteratively solve a convex optimization problem in each iteration. The simulation results are provided to investigate the CR sum-rate (spectral efficiency) performance and the robustness against the CSI uncertainty.
\end{abstract}

Keywords- Cognitive Radio, Full-Duplex, Multi-user MIMO, Robust Precoding Design, Imperfect CSI.

\section{INTRODUCTION}

The substantial spectral efficiency (SE) improvement of full-duplex (FD) transmission modes as compared to traditional half-duplex counterparts has been demonstrated in FD multi-user multiple-input multiple-output (MU-MIMO) cellular networks [1-4]. The sum rate maximization (SRM) algorithm based on the sequential convex programming (SCP) approach was proposed for a single cellular FD MU-MIMO network without and with the impact of co-channel interference (CCI) in [1] and [2], respectively. Reference [3] jointly designed precoding matrices for bidirectional FD interference channel (IC) systems by minimizing the system sum mean squared error (MSE), and then expanded this algorithm for FD cellular systems. Alternatively, reference [4] proposed a novel SCP for multicell FD MU-MIMO, namely successive quadratic convex programming (SQCP). However, almost all above works adopted the assumption of perfect channel state information (CSI) knowledge at transceivers. This assumption is difficult to achieve in practice. Hence, to be more precise on practical conditions of wireless communication systems, the CSI errors are taken into consideration for single cell and multi-cell FD MU-MIMO systems in [5, 6].

Along with FD techniques, cognitive radio (CR) approaches have attracted an extensive consideration [712] since they can efficiently reuse spectral resources. Reference [7] developed a robust linear transceiver design algorithm for ad hoc cognitive network based on the MSE minimization under imperfect CSIs with both uncertain models, namely norm bounded error (NBE) and stochastic error (SE). Reference [8] designed the transmit covariance matrices and receive filters to optimize the MSE performance under CSI inaccuracy assumptions. On the other hand, the interference alignment (IA) based algorithms were developed for multiple pair of SUs and PUs to minimize the system MSE or maximize the network energy efficiency in [9] and [10], respectively. Alternatively, in the scenario of the cognitive MU-MIMO downlink model, reference [11] used zero-forcing methods to cancel inter-user interferences and, then, developed a barrier interior-point method to optimize the system throughput under transmit per-antenna power and interference temperature constraints. Reference [12] introduced an iterative quadratic convex algorithm to maximize the sum-rate by jointly designing beamformers and antenna selection for secondary quality of service requirements in multiple-input single-output (MISO) broadcast channels. More recently, the combination of both FD transmission and $\mathrm{CR}$ techniques was studied in $[13,14]$. The authors in [13] considered the design problems based on minimum sum MSE or minimummaximum MSE for CR FD MU-MIMO systems. Reference [14] addressed two robust algorithms based on the MSE under the channel uncertainties.

Motivated from above works, this paper considers an underlay FD MU-MIMO secondary system operating within the multiple multi-antenna PU coverage as $[13,14]$. The CSI knowledge of all links in the secondary FD network is assumed to be perfectly known, however 
the CSI of the links from SUs to PUs are imperfectly known. Additionally, the NBE model is used to capture the SUs-PUs channel uncertainties [8]. Different from the works in $[13,14]$ which are based on the MSE minimization, the present paper aims at maximizing the spectral efficiency of the CR network. More specifically, the transmit precoder matrices at both base station (BS) and uplink users in the secondary network are jointly designed to maximize the sum rate (i.e., spectral efficiency) of the secondary system under the constraints of the harmful interference caused to the PUs. The considered robust design is an intractable optimization problem due to the non-concavity of the achievable rates along with robust interference semiinfinite constraints. Thus, it is difficult to directly find the optimal points. In this paper, by making the use of the fact that the objective function can be expressed as the difference of two concave (d.c.) ones, the sumrate objective function can be lower-bounded by a concave function $[1,2,15]$. On the other hand, to cope with the semi-infinite robust interference constraints, the S-procedure is used to transform the interference constraints into linear matrix inequality (LMI) ones. As a result, an iterative algorithm is derived to find the optimal precodes by solving the convex optimization in each iteration. The numerical results are conducted to evaluate the convergence behaviors of the iterative algorithm and to investigate the system sum-rate performance and robustness of the designed precoders.

The rest of this paper is organized as follows. Section 2 introduces the signal and system models and the assumptions considered in the paper. Section 3 presents the design problem for maximizing the system sum-rate and describes the iterative optimization algorithm. Finally, the simulation results and conclusions are provided in Section 4 and Section 5, respectively.

Notations: Bold lower and upper case letters represent vectors and matrices, respectively. $\mathbf{Y}^{\dagger}, \operatorname{Tr}(\mathbf{Y})$ and $|\mathbf{Y}|$ are the Hermitian, trace and determinant operators of $\mathbf{Y}$, respectively. $\mathbf{Y} \succcurlyeq 0$ means that $\mathbf{Y}$ is a positive semidefinite matrix and $\mathbf{I}_{n}$ represents $n \times n$ identity matrix. The notation $\mathbf{Y} \otimes \mathbf{M}$ refers to the Kronecker product of $\mathbf{Y}$ and $\mathbf{M} . \Re\{\cdot\}$ returns the real values. The $\operatorname{vec}(\mathbf{M})$ function denotes the vectorization of $\mathbf{M}$.

\section{System Model}

Consider an FD MU-MIMO CR network as illustrated in Figure 1 where one secondary system is sharing the spectrum with $P$ primary users (PUs). In the secondary network, one FD base station (BS), denoted by $\mathcal{B}$, is enable to simultaneously communicate with $U$ uplink (UL) users (ULUs) in UL channels, and $D$ downlink (DL) users (DLUs) in DL channels. The set of ULUs, DLUs, PUs are denoted by $\mathcal{U}=\left\{1_{\mathrm{u}}, 2_{\mathrm{u}}, \ldots, U_{\mathrm{u}}\right\}, \mathcal{D}=$ $\left\{1_{\mathrm{d}}, 2_{\mathrm{d}}, \ldots, D_{\mathrm{d}}\right\}$, and $\mathcal{V}=\left\{1_{\mathrm{p}}, 2_{\mathrm{p}}, \ldots, P_{\mathrm{p}}\right\}$ respectively. Base station $\mathcal{B}$ is equipped with $N_{\mathcal{B}}$ receive antennas for uplink transmission and $M_{\mathcal{B}}$ transmit antennas for downlink transmission, while ULU $\ell_{\mathrm{u}}$ and DLU $k_{\mathrm{d}}$ have $M_{\ell_{\mathrm{u}}}$ and $N_{k_{\mathrm{d}}}$ antennas, respectively. PU $n_{\mathrm{p}}$ is equipped

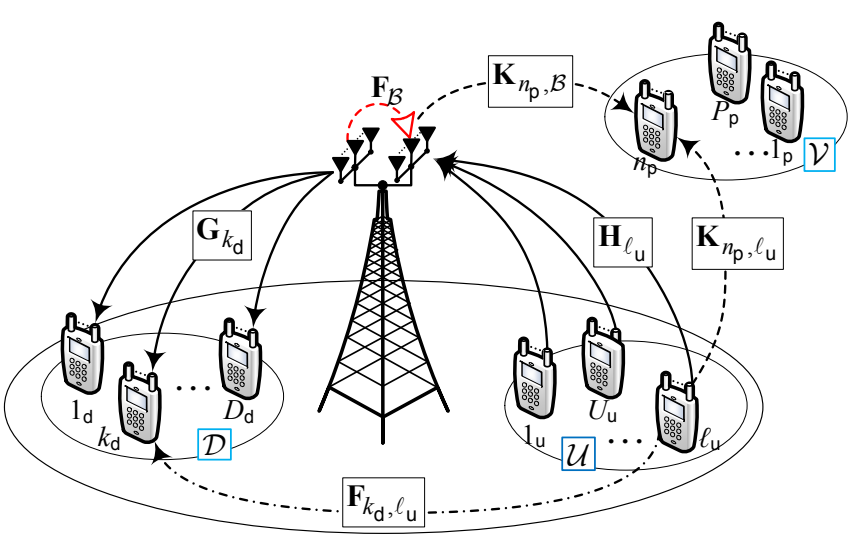

Figure 1: The FD MU-MIMO underlay spectrum sharing cognitive system.

$L_{n_{\mathrm{p}}}$ receive antennas. Throughout this paper, we use $k_{\mathrm{d}} \in \mathcal{D}, \ell_{\mathrm{u}} \in \mathcal{U}$ and $n_{\mathrm{p}} \in \mathcal{V}$ to refer to the $k$-th user in the DL channels, the $\ell$-th user in the UL channels and the $n$-th user in the set of PUs, respectively.

\subsection{Signal Model}

Let $\mathbf{s}_{k_{\mathrm{d}}} \in \mathbb{C}^{d_{k_{\mathrm{d}}} \times 1}, \mathbf{s}_{\ell_{\mathrm{u}}} \in \mathbb{C}^{d_{\ell_{\mathrm{u}}} \times 1}$ be the vectors of symbols intended to DLU $k_{\mathrm{d}}$ and from ULU $\ell_{\mathrm{u}}$, where $d_{k_{\mathrm{d}}}$ and $d_{\ell_{\mathrm{u}}}$, satisfying $d_{k_{\mathrm{d}}} \leq \min \left(N_{k_{\mathrm{d}}}, M_{\mathcal{B}}\right)$, $d_{\ell_{u}} \leq \min \left(M_{\ell_{u}}, N_{\mathcal{B}}\right)$, are the numbers of data streams associated with users $k_{\mathrm{d}}$ and $\ell_{\mathrm{u}}$, respectively.

In UL channels, the received vector signal at $\mathcal{B}$, i.e. $\mathbf{y}_{\mathcal{B}} \in \mathbb{C}^{N_{\mathcal{B}} \times 1}$, is given by

$$
\mathbf{y}_{\mathcal{B}}=\underbrace{\sum_{\ell_{\mathrm{u}} \in \mathcal{U}} \mathbf{H}_{\ell_{\mathrm{u}}} \mathbf{A}_{\ell_{\mathrm{u}}} \mathbf{s}_{\ell_{\mathrm{u}}}}_{\text {Desired signals }}+\underbrace{\sum_{k_{\mathrm{d}} \in \mathcal{D}} \mathbf{F}_{\mathcal{B}} \mathbf{B}_{k_{\mathrm{d}}} \mathbf{s}_{k_{\mathrm{d}}}}_{\text {Self-Interference }}+\underbrace{\mathbf{n}_{\mathcal{B}}}_{\text {Noise }},
$$

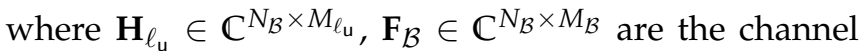
gains of $\ell_{\mathrm{u}} \rightarrow \mathcal{B}$ link and SI link from transmit to receive antennas at the $\mathrm{BS}$, respectively. Furthermore, $\mathbf{A}_{\ell_{\mathrm{u}}} \in \mathbb{C}^{M_{\ell_{\mathrm{u}}} \times d_{\ell_{\mathrm{u}}}}, \mathbf{B}_{k_{\mathrm{d}}} \in \mathbb{C}^{M_{\mathcal{B}} \times d_{k_{\mathrm{d}}}}$ denote precoding matrices for user $\ell_{\mathrm{u}}, k_{\mathrm{d}}$, respectively. $\mathbf{n}_{\mathcal{B}} \in \mathbb{C}^{N_{\mathcal{B}} \times 1}$ refers to the additive white Gaussian noise (AWGN) at $\mathcal{B}$ with zero mean and covariance matrix $\sigma_{\mathcal{B}}^{2} \mathbf{I}_{N_{\mathcal{B}}}$.

In addition, the received signal at DLU $k_{\mathrm{d}}$, denoted by $\mathbf{y}_{k_{\mathrm{d}}} \in \mathbb{C}^{N_{k_{\mathrm{d}}} \times 1}$, can be expressed as

$$
\begin{aligned}
\mathbf{y}_{k_{\mathrm{d}}}= & \underbrace{\mathbf{G}_{k_{\mathrm{d}}} \mathbf{B}_{k_{\mathrm{d}}} \mathbf{s}_{k_{\mathrm{d}}}}_{\text {Desired signals }}+\underbrace{\sum_{i_{\mathrm{d}} \in \mathcal{D} \backslash\left\{k_{\mathrm{d}}\right\}} \mathbf{G}_{k_{\mathrm{d}}} \mathbf{B}_{i_{\mathrm{d}}} \mathbf{s}_{i_{\mathrm{d}}}}_{\text {Multi-User Interference }} \\
& +\underbrace{\sum_{t_{\mathrm{u}} \in \mathcal{U}} \mathbf{F}_{k_{\mathrm{d}}, t_{\mathrm{u}}} \mathbf{A}_{t_{\mathrm{u}}} \mathbf{s}_{t_{\mathrm{u}}}}_{\text {Co-channel Interference }}+\underbrace{\mathbf{n}_{k_{\mathrm{d}}}}_{\text {Noise }}
\end{aligned}
$$

where $\mathbf{F}_{k_{\mathrm{d}}, t_{\mathrm{u}}} \in \mathbb{C}^{N_{k_{\mathrm{d}}} \times M_{t_{\mathrm{u}}}}$ represents the CCI channel coefficient at $k_{\mathrm{d}}$ from ULU $t_{\mathrm{u}}$, and $\mathbf{n}_{k_{\mathrm{d}}} \in \mathbb{C}^{N_{k_{\mathrm{d}}} \times 1}$ represents the AWGN at $k_{\mathrm{d}}$ with zero mean and covariance matrix $\sigma_{k_{\mathrm{d}}}^{2} \mathbf{I}_{N_{k_{\mathrm{d}}}}$.

Define $\mathbf{Q}_{k_{\mathrm{d}}} \triangleq \mathbf{B}_{k_{\mathrm{d}}} \mathbf{B}_{k_{\mathrm{d}}}^{\dagger}$ and $\mathbf{R}_{\ell_{\mathrm{u}}} \triangleq \mathbf{A}_{\ell_{\mathrm{u}}} \mathbf{A}_{\ell_{\mathrm{u}}}^{\dagger}$ which are the transmit covariance matrices associated with the $k_{\mathrm{d}}$-th DLU and $\ell_{\mathrm{u}}$-th ULU, respectively. For notation simplicity, we define $\mathbf{Q}_{\mathrm{D}} \triangleq\left\{\mathbf{Q}_{k_{\mathrm{d}}} \mid k_{\mathrm{d}} \in \mathcal{D}\right\}$ and $\mathbf{R}_{\mathrm{U}} \triangleq$ $\left\{\mathbf{R}_{\ell_{\mathrm{u}}} \mid \ell_{\mathrm{u}} \in \mathcal{U}\right\}$ are the sets of DL and UL covariance 
matrices, respectively. Thus, the achievable data rate of user $k_{d}$ in the DL channel can be defined by

$$
R_{k_{\mathrm{d}}}\left(\mathbf{Q}_{\mathrm{D}}, \mathbf{R}_{\mathrm{U}}\right)=\log _{2}\left|\mathbf{I}_{N_{k_{\mathrm{d}}}}+\left(\sigma_{k_{\mathrm{d}}}^{2} \mathbf{I}_{N_{k_{\mathrm{d}}}}+\mathbf{\Omega}_{k_{\mathrm{d}}}\right)^{-1} \mathbf{C}_{k_{\mathrm{d}}}\right|,
$$

where $\mathbf{C}_{k_{\mathrm{d}}} \triangleq \mathbf{G}_{k_{\mathrm{d}}} \mathbf{Q}_{k_{\mathrm{d}}} \mathbf{G}_{k_{\mathrm{d}}}^{+}$denotes the covariance matrix of desired signal and $\boldsymbol{\Omega}_{k_{\mathrm{d}}} \triangleq \sum_{i_{\mathrm{d}} \in \mathcal{D} \backslash\left\{k_{\mathrm{d}}\right\}} \mathbf{G}_{k_{\mathrm{d}}} \mathbf{Q}_{i_{\mathrm{d}}} \mathbf{G}_{k_{\mathrm{d}}}^{+}+$ $\sum_{t_{\mathrm{u}} \in \mathcal{U}} \mathbf{F}_{k_{\mathrm{d}}, t_{\mathrm{u}}} \mathbf{R}_{t_{\mathrm{u}}} \mathbf{F}_{k_{\mathrm{d}}, t_{\mathrm{u}}}^{+}$is the covariance matrix of the multi-user plus co-channel interferences of $k_{\mathrm{d}}$.

Furthermore, from (1) the total achievable rate of UL channels can be formulated as

$$
R_{\mathcal{B}}\left(\mathbf{Q}_{\mathrm{D}}, \mathbf{R}_{\mathrm{U}}\right)=\log _{2}\left|\mathbf{I}_{N_{\mathcal{B}}}+\left(\sigma_{\mathcal{B}}^{2} \mathbf{I}_{N_{\mathcal{B}}}+\mathbf{\Omega}_{\mathcal{B}}\right)^{-1} \mathbf{C}_{\mathcal{B}}\right|,
$$

where $\boldsymbol{\Omega}_{\mathcal{B}} \triangleq \sum_{k_{\mathrm{d}} \in \mathcal{D}} \mathbf{F}_{\mathcal{B}} \mathbf{Q}_{k_{\mathrm{d}}} \mathbf{F}_{\mathcal{B}}^{+}, \mathbf{C}_{\mathcal{B}} \triangleq \sum_{\ell_{\mathrm{u}} \in \mathcal{U}} \mathbf{H}_{\ell_{\mathrm{u}}} \mathbf{R}_{\ell_{\mathrm{u}}} \mathbf{H}_{\ell_{\mathrm{u}}}^{+}$ are covariance matrices of SI and desired signal at BS respectively.

\subsection{Robust Interference Power Constraints}

Concerning interference at PU devices, the covariance matrix of the received signals at PU $n_{\mathrm{p}}$ with $n_{\mathrm{p}} \in \mathcal{V}$ is given by

$$
\mathbf{C}_{n_{\mathrm{p}}}=\sum_{\ell_{\mathrm{u}} \in \mathcal{U}} \mathbf{K}_{n_{\mathrm{p}}, \ell_{\mathrm{u}}} \mathbf{R}_{\ell_{\mathrm{u}}} \mathbf{K}_{n_{\mathrm{p}}, \ell_{\mathrm{u}}}^{\dagger}+\mathbf{K}_{n_{\mathrm{p}}, \mathcal{B}} \mathbf{Q}_{k_{\mathrm{d}}}^{\text {sum }} \mathbf{K}_{n_{\mathrm{p}}, \mathcal{B}^{\prime}}^{+}
$$

where $\mathbf{K}_{n_{\mathrm{p}}, \ell_{\mathrm{u}}} \in \mathbb{C}^{L_{n_{\mathrm{p}}} \times M_{\ell_{\mathrm{u}}}}, \mathbf{K}_{n_{\mathrm{p}}, \mathcal{B}} \in \mathbb{C}^{L_{n_{\mathrm{p}}} \times M_{\mathcal{B}}}$ are the channel coefficients at $n_{\mathrm{p}}$ from $\ell_{\mathbf{u}}$ and $\mathcal{B}$, respectively while we have defined $\mathbf{Q}_{k_{\mathrm{d}}}^{\text {sum }} \triangleq \sum_{k_{\mathrm{d}} \in \mathcal{D}} \mathbf{Q}_{k_{\mathrm{d}}}$. Hence, the total interference power at PU $n_{\mathrm{p}}$ can be given by

$$
I_{n_{\mathrm{p}}}\left(\mathbf{Q}_{\mathrm{D}}, \mathbf{R}_{\mathrm{U}}\right)=\operatorname{Tr}\left(\mathbf{C}_{n_{\mathrm{p}}}\right)
$$

In the considered system, the PUs have higher priority to access the spectral resource than the SUs. Consequently, the FD MU-MIMO CR network is designed to guarantee that the total interference power at each PU caused from the CR system is not greater than a predetermined allowable threshold. Supposing that the allowable interference threshold at PU $n_{\mathrm{p}}$ is $\gamma_{n_{\mathrm{p}}}$, the interference constraints can be formulated by

$$
I_{n_{\mathrm{p}}}\left(\mathbf{Q}_{\mathrm{D}}, \mathbf{R}_{\mathrm{U}}\right) \leq \gamma_{n_{\mathrm{p}}}, \forall n_{\mathrm{p}} \in \mathcal{V}
$$

However, by the reason of not full cooperation between the FD cognitive MU-MIMO and PU systems, the channel gains between these links cannot be perfectly known [8]. Thus, the robust interference power constraints under imperfect known CSI is expressed as

$$
\begin{aligned}
I_{n_{\mathrm{p}}}\left(\mathbf{Q}_{\mathrm{D}}, \mathbf{R}_{\mathrm{U}}\right) \leq \gamma_{n_{\mathrm{p}}}, & \forall \mathbf{K}_{n_{\mathrm{p}}, \mathcal{B}} \in \mathcal{K}_{n_{\mathrm{p}}, \mathcal{B}}^{\mathrm{NBE}}, \\
& \forall \mathbf{K}_{n_{\mathrm{p}}, \ell_{\mathrm{u}}} \in \mathcal{K}_{n_{\mathrm{p}}, \ell_{\mathrm{u}}}^{\mathrm{NBE}} \\
& \forall n_{\mathrm{p}} \in \mathcal{V}, \forall \ell_{\mathbf{u}} \in \mathcal{U},
\end{aligned}
$$

where $\mathcal{K}_{n_{\mathrm{p}}, \mathcal{B}}^{\mathrm{NBE}}$ and $\mathcal{K}_{n_{\mathrm{p}}, \ell_{\mathrm{u}}}^{\mathrm{NBE}}$ are probable sets of inaccuracy CSI. In this paper, the imperfect CSI errors are modelled by norm-bounded error (NBE) models [5-7, 14]

$$
\begin{aligned}
& \mathcal{K}_{n_{\mathrm{p}}, \ell_{\mathrm{u}}}^{\mathrm{NBE}} \triangleq\left\{\hat{\mathbf{K}}_{n_{\mathrm{p}}, \ell_{\mathrm{u}}}+\boldsymbol{\Delta}_{n_{\mathrm{p}}, \ell_{\mathrm{u}}}:\left\|\boldsymbol{\Delta}_{n_{\mathrm{p}}, \ell_{\mathrm{u}}}\right\|_{F} \leq \varepsilon_{n_{\mathrm{p}}, \ell_{\mathrm{u}}}\right\}, \\
& \mathcal{K}_{n_{\mathrm{p}}, \mathcal{B}}^{\mathrm{NBE}} \triangleq\left\{\hat{\mathbf{K}}_{n_{\mathrm{p}}, \mathcal{B}}+\boldsymbol{\Delta}_{n_{\mathrm{p}}, \mathcal{B}}:\left\|\boldsymbol{\Delta}_{n_{\mathrm{p}}, \mathcal{B}}\right\|_{F} \leq \varepsilon_{n_{\mathrm{p}}, \mathcal{B}}\right\} .
\end{aligned}
$$

Here, $\hat{\mathbf{K}}_{n_{\mathrm{p}}, \ell_{\mathrm{u}}}$ and $\hat{\mathbf{K}}_{n_{\mathrm{p}}, \mathcal{B}}$ are estimated channels. $\boldsymbol{\Delta}_{n_{\mathrm{p}}, \ell_{\mathrm{u}}}$ and $\boldsymbol{\Delta}_{n_{\mathrm{p}}, \mathcal{B}}$ denote channel error matrices. $\varepsilon_{n_{\mathrm{p}}, \ell_{\mathrm{u}}}$ and $\varepsilon_{n_{\mathrm{p}}, \mathcal{B}}$ represent the uncertainty bounds.

\section{Robust Precoding Designs for Sum Rate Maximization OF FD CR MU-MIMO SySTEM}

This section aims at finding the optimal precoding matrices to maximize the sum rate under the constraints of per user power constraint (PUPC) at each UL device, sum power constraint (SPC) at $\mathcal{B}$ and the interference constraint at each PU.

\subsection{Sum Rate Maximization Problem}

The overall system achievable rate is given by

$$
R_{\mathrm{Tot}}\left(\mathbf{Q}_{\mathrm{D}}, \mathbf{R}_{\mathrm{U}}\right) \triangleq R_{\mathcal{B}}\left(\mathbf{Q}_{\mathrm{D}}, \mathbf{R}_{\mathrm{U}}\right)+\sum_{k_{\mathrm{d}} \in \mathcal{D}} R_{k_{\mathrm{d}}}\left(\mathbf{Q}_{\mathrm{D}}, \mathbf{R}_{\mathrm{U}}\right)
$$

The robust sum-rate maximization (SRM) design problem subject to the transmit power constraints and interference constraints under the imperfect CSI of links from $\mathcal{B}$ and ULUs to $n_{\mathrm{p}}$ is mathematically formulated as

$$
\begin{aligned}
\max _{\left\{\mathbf{Q}_{\mathrm{D}}, \mathbf{R}_{\mathrm{U}}\right\}_{\succcurlyeq 0}} & R_{\operatorname{Tot}}\left(\mathbf{Q}_{\mathrm{D}}, \mathbf{R}_{\mathrm{U}}\right) \\
\text { s.t. } & \operatorname{Tr}\left(\mathbf{R}_{\ell_{\mathrm{u}}}\right) \leq P_{\ell_{\mathrm{u}}, \max }, \forall \ell_{\mathbf{u}} \in \mathcal{U}, \\
& \sum_{k_{\mathrm{d}} \in \mathcal{D}} \operatorname{Tr}\left(\mathbf{Q}_{k_{\mathrm{d}}}\right) \leq P_{\mathcal{B}, \text { max }},
\end{aligned}
$$

(8),

where $P_{\ell_{\mathrm{u}}, \max }, P_{\mathcal{B}, \max }, \gamma_{n_{\mathrm{p}}}$ are the maximum transmit power at UL $\ell_{u}$, total maximum transmit power at BS and the maximum allowable interference power at PU $n_{\mathrm{p}}$, respectively. Note that $(12 \mathrm{~b})$ imposes the PUPC at each UL user, (12c) is the SPC at $\mathcal{B}$, and (12d) represents the robust interference power constraints to each PU.

\subsection{Difference of Two Concave Functions}

As can be seen from problem (12), the objective function is a non-concave which makes the optimization problem intractable. Thus, we derive an algorithm based the SCP approach to handle this challenging non-convex problem. The key idea of the SCP method is to iteratively find the optimal solution of a convex problem in each iteration. Therefore, in the sequel, we will derive an alternative problem by approximating the objective function into a concave one. Exploiting the concavity of logdet function, one has [16, p.69], [1, 2, 15]

$$
\begin{aligned}
\log _{2}|\mathbf{M}+\mathbf{Y}| \leq & \log _{2}\left|\mathbf{M}+\mathbf{Y}_{0}\right| \\
& +\frac{1}{\ln (2)} \operatorname{Tr}\left[\left(\mathbf{M}+\mathbf{Y}_{0}\right)^{-1}\left(\mathbf{Y}-\mathbf{Y}_{0}\right)\right]
\end{aligned}
$$

for any $\mathbf{M}, \mathbf{Y}, \mathbf{Y}_{0} \succcurlyeq 0$ and the equality is achieved when $\mathbf{Y}_{0}=\mathbf{Y}$. Then, we can rewrite the achievable rate at DLU $k_{\mathrm{d}}$ in (3) as

$$
\begin{aligned}
R_{k_{\mathrm{d}}}\left(\mathbf{Q}_{\mathrm{D}}, \mathbf{R}_{\mathrm{U}}\right)= & \log _{2}\left|\sigma_{k_{\mathrm{d}}}^{2} \mathbf{I}_{N_{k_{\mathrm{d}}}}+\mathbf{\Omega}_{k_{\mathrm{d}}}+\mathbf{C}_{k_{\mathrm{d}}}\right| \\
& -\log _{2}\left|\sigma_{k_{\mathrm{d}}}^{2} \mathbf{I}_{N_{k_{\mathrm{d}}}}+\mathbf{\Omega}_{k_{\mathrm{d}}}\right|,
\end{aligned}
$$


and the total $\mathrm{UL}$ rate at $\mathcal{B}(4)$ as

$$
\begin{aligned}
R_{\mathcal{B}}\left(\mathbf{Q}_{\mathrm{D}}, \mathbf{R}_{\mathrm{U}}\right)= & \log _{2}\left|\sigma_{\mathcal{B}}^{2} \mathbf{I}_{N_{\mathcal{B}}}+\mathbf{\Omega}_{\mathcal{B}}+\mathbf{C}_{\mathcal{B}}\right| \\
& -\log _{2}\left|\sigma_{\mathcal{B}}^{2} \mathbf{I}_{N_{\mathcal{B}}}+\mathbf{\Omega}_{\mathcal{B}}\right| .
\end{aligned}
$$

It is worth noting that both (14) and (15) are the difference of two concave functions. Hence, by applying (13) on the second term of right hand side in (14), the approximation of $k_{\mathrm{d}}$ DLU rate at a feasible set of $\mathcal{F}^{(\kappa)} \triangleq$ $\left\{\mathbf{Q}_{\ell_{\mathrm{u}}}^{(\kappa)}, \mathbf{R}_{k_{\mathrm{d}}}^{(\kappa)} \mid k_{\mathrm{d}} \in \mathcal{D}, \ell_{\mathbf{u}} \in \mathcal{U}\right\}$ can be formulated as

$$
\begin{aligned}
& R_{k_{\mathrm{d}}}\left(\mathbf{Q}_{\mathrm{D}}, \mathbf{R}_{\mathrm{U}}\right) \geq \phi_{k_{\mathrm{d}}}\left(\mathbf{Q}_{\mathrm{D}}, \mathbf{R}_{\mathrm{U}}\right)=-\log _{2}\left|\mathbf{\Phi}_{k_{\mathrm{d}}}^{(\kappa)}\right| \\
& +\log _{2}\left|\sigma_{k_{\mathrm{d}}}^{2} \mathbf{I}_{N_{k_{\mathrm{d}}}}+\sum_{i_{\mathrm{d}} \in \mathcal{D}} \mathbf{G}_{k_{\mathrm{d}}} \mathbf{Q}_{i_{\mathrm{d}}} \mathbf{G}_{k_{\mathrm{d}}}^{+}+\sum_{\ell_{\mathrm{u}} \in \mathcal{U}} \mathbf{F}_{k_{\mathrm{d}}, \ell_{\mathrm{u}}} \mathbf{R}_{\ell_{\mathrm{u}}} \mathbf{F}_{k_{\mathrm{d}}, \ell_{\mathrm{u}}}^{+}\right| \\
& -\frac{1}{\ln (2)} \operatorname{Tr}\left[\left(\boldsymbol{\Phi}_{k_{\mathrm{d}}}^{(\kappa)}\right)^{-1} \sum_{i_{\mathrm{d}} \in \mathcal{D} \backslash\left\{k_{\mathrm{d}}\right\}} \mathbf{G}_{k_{\mathrm{d}}}\left(\mathbf{Q}_{i_{\mathrm{d}}}-\mathbf{Q}_{i_{\mathrm{d}}}^{(\kappa)}\right) \mathbf{G}_{k_{\mathrm{d}}}^{+}\right] \\
& -\frac{1}{\ln (2)} \operatorname{Tr}\left[\left(\boldsymbol{\Phi}_{k_{\mathrm{d}}}^{(\kappa)}\right)^{-1} \sum_{\ell_{\mathrm{u}} \in \mathcal{U}} \mathbf{F}_{k_{\mathrm{d}}, \ell_{\mathrm{u}}}\left(\mathbf{R}_{\ell_{\mathrm{u}}}-\mathbf{R}_{\ell_{\mathrm{u}}}^{(\kappa)}\right) \mathbf{F}_{k_{\mathrm{d}}, \ell_{\mathrm{u}}}^{+}\right],
\end{aligned}
$$

where $\boldsymbol{\Phi}_{k_{\mathrm{d}}}^{(\kappa)} \triangleq \sigma_{k_{\mathrm{d}}}^{2} \mathbf{I}_{N_{k_{\mathrm{d}}}}+\sum_{i_{\mathrm{d}} \in \mathcal{D} \backslash\left\{k_{\mathrm{d}}\right\}} \mathbf{G}_{k_{\mathrm{d}}} \mathbf{Q}_{i_{\mathrm{d}}}^{(\kappa)} \mathbf{G}_{k_{\mathrm{d}}}^{+}+$ $\sum_{\ell_{\mathrm{u}} \in \mathcal{U}} \mathbf{F}_{k_{\mathrm{d}}, \ell_{\mathrm{u}}} \mathbf{R}_{\ell_{\mathrm{u}}}^{(\kappa)} \mathbf{F}_{k_{\mathrm{d}}, \ell_{\mathrm{u}}}^{+}$.

Similarly, the lower bound of the UL sum rate in (15) can be formulated at $\mathcal{F}^{(\kappa)}$ as

$$
\begin{aligned}
& R_{\mathcal{B}}\left(\mathbf{Q}_{\mathrm{D}}, \mathbf{R}_{\mathrm{U}}\right) \geq \phi_{\mathcal{B}}\left(\mathbf{Q}_{\mathrm{D}}, \mathbf{R}_{\mathrm{U}}\right)=-\log _{2}\left|\mathbf{\Phi}_{\mathcal{B}}^{(\kappa)}\right| \\
& \quad+\log _{2}\left|\sigma_{\mathcal{B}}^{2} \mathbf{I}_{N_{\mathcal{B}}}+\sum_{k_{\mathrm{d}} \in \mathcal{D}} \mathbf{F}_{\mathcal{B}} \mathbf{Q}_{k_{\mathrm{d}}} \mathbf{F}_{\mathcal{B}}^{+}+\sum_{\ell_{\mathrm{u}} \in \mathcal{U}} \mathbf{H}_{\ell_{\mathrm{u}}} \mathbf{R}_{\ell_{\mathrm{u}}} \mathbf{H}_{\ell_{\mathrm{u}}}^{\dagger}\right| \\
& \quad-\frac{1}{\ln (2)} \operatorname{Tr}\left[\left(\mathbf{\Phi}_{\mathcal{B}}^{(\kappa)}\right)^{-1} \sum_{k_{\mathrm{d}} \in \mathcal{D}} \mathbf{F}_{\mathcal{B}}\left(\mathbf{Q}_{k_{\mathrm{d}}}-\mathbf{Q}_{k_{\mathrm{d}}}^{(\kappa)}\right) \mathbf{F}_{\mathcal{B}}^{\dagger}\right],
\end{aligned}
$$

with $\boldsymbol{\Phi}_{\mathcal{B}}^{(\kappa)} \triangleq \sigma_{\mathcal{B}}^{2} \mathbf{I}_{N_{\mathcal{B}}}+\sum_{k_{\mathrm{d}} \in \mathcal{D}} \mathbf{F}_{\mathcal{B}} \mathbf{Q}_{k_{\mathrm{d}}}^{(\kappa)} \mathbf{F}_{\mathcal{B}}^{\dagger}$. Therefore, the total sum rate is tightly lower bounded by

$$
\begin{aligned}
\phi_{\mathrm{Tot}}\left(\mathbf{Q}_{\mathrm{D}}, \mathbf{R}_{\mathrm{U}}\right) \triangleq & \phi_{\mathcal{B}}\left(\mathbf{Q}_{\mathrm{D}}, \mathbf{R}_{\mathrm{U}}\right)+\sum_{k_{\mathrm{d}} \in \mathcal{D}} \phi_{k_{\mathrm{d}}}\left(\mathbf{Q}_{\mathrm{D}}, \mathbf{R}_{\mathrm{U}}\right) \\
& \leq R_{\mathrm{Tot}}\left(\mathbf{Q}_{\mathrm{D}}, \mathbf{R}_{\mathrm{U}}\right) .
\end{aligned}
$$

It is a concave function with respect to $\left\{\mathbf{Q}_{\mathrm{D}}, \mathbf{R}_{\mathrm{U}}\right\}$. Therefore, the SRM problem (12) can be rewritten as a convex one in iteration $\kappa$ as

$$
\begin{aligned}
\max _{\{\mathbf{Q}\} \succcurlyeq 0} & \phi_{\text {Tot }}\left(\mathbf{Q}_{\mathrm{D}}, \mathbf{R}_{\mathrm{U}}\right) \\
\text { s.t. } & (12 \mathrm{~b}),(12 \mathrm{c}),(12 \mathrm{~d}) .
\end{aligned}
$$

Although the objective function now is convex, the interference power constraints $(12 \mathrm{~d})$ is quite intractable to handle since they are semi-infinite constraints.

\subsection{Linear Matrix Inequality (LMI) Transformation for Robust PU Interference Power Constraints}

By introducing the auxiliary variables $\gamma_{n_{\mathrm{p}}, \ell_{\mathrm{u}}}$, and $\gamma_{n_{\mathrm{p}}, \mathcal{B}}$ with $\ell_{\mathbf{u}} \in \mathcal{U}, n_{\mathrm{p}} \in \mathcal{V}$, the interference con- straints (8) can be recast as

$$
\begin{aligned}
& \sum_{\ell_{\mathrm{u}} \in \mathcal{U}} \gamma_{n_{\mathrm{p}}, \ell_{\mathrm{u}}}+\gamma_{n_{\mathrm{p}}, \mathcal{B}} \leq \gamma_{n_{\mathrm{p}}}, \forall n_{\mathrm{p}} \in \mathcal{V}, \\
& \quad \gamma_{n_{\mathrm{p}}, \ell_{\mathrm{u}}} \geq 0, \gamma_{n_{\mathrm{p}}, \mathcal{B}} \geq 0, \forall \ell_{\mathrm{u}} \in \mathcal{U}, \\
& \operatorname{Tr}\left(\mathbf{K}_{n_{\mathrm{p}}, \ell_{\mathrm{u}}} \mathbf{R}_{\ell_{\mathrm{u}}} \mathbf{K}_{n_{\mathrm{p}}, \ell_{\mathrm{u}}}^{\dagger}\right) \leq \gamma_{n_{\mathrm{p}}, \ell_{\mathrm{u}}}, \forall \mathbf{K}_{n_{\mathrm{p}}, \ell_{\mathrm{u}}} \in \mathcal{K}_{n_{\mathrm{p}}, \ell_{\mathrm{u}}}^{\mathrm{NBE}} \\
& \operatorname{Tr}\left(\mathbf{K}_{n_{\mathrm{p}}, \mathcal{B}} \mathbf{Q}_{k_{\mathrm{d}}}^{\mathrm{sum}} \mathbf{K}_{n_{\mathrm{p}}, \mathcal{B}}^{+}\right) \leq \gamma_{n_{\mathrm{p}}, \mathcal{B}}, \forall \mathbf{K}_{n_{\mathrm{p}}, \mathcal{B}} \in \mathcal{K}_{n_{\mathrm{p}}, \mathcal{B}}^{\mathrm{NBE}} .
\end{aligned}
$$

Then, we define the term $\operatorname{Tr}\left(\mathbf{K}_{n_{\mathrm{p}}, \ell_{\mathrm{u}}} \mathbf{R}_{\ell_{\mathrm{u}}} \mathbf{K}_{n_{\mathrm{p}}, \ell_{\mathrm{u}}}^{\dagger}\right) \triangleq \beta_{n_{\mathrm{p}}, \ell_{\mathrm{u}}}$ in (20c) and substitute $\mathbf{K}_{n_{\mathrm{p}}, \ell_{\mathrm{u}}}=\hat{\mathbf{K}}_{n_{\mathrm{p}}, \ell_{\mathrm{u}}}+\boldsymbol{\Delta}_{n_{\mathrm{p}}, \ell_{\mathrm{u}}}$ to yield

$$
\begin{aligned}
\beta_{n_{\mathrm{p}}, \ell_{\mathrm{u}}}= & \operatorname{Tr}\left(\hat{\mathbf{K}}_{n_{\mathrm{p}}, \ell_{\mathrm{u}}} \mathbf{R}_{\ell_{\mathrm{u}}} \hat{\mathbf{K}}_{n_{\mathrm{p}}, \ell_{\mathrm{u}}}^{+}\right)+2 \Re\left[\operatorname{Tr}\left(\hat{\mathbf{K}}_{n_{\mathrm{p}}, \ell_{\mathrm{u}}} \mathbf{R}_{\ell_{\mathrm{u}}} \Delta_{n_{\mathrm{p}}, \ell_{\mathrm{u}}}^{+}\right)\right] \\
& +\operatorname{Tr}\left(\boldsymbol{\Delta}_{n_{\mathrm{p}}, \ell_{\mathrm{u}}} \mathbf{R}_{\ell_{\mathrm{u}}} \Delta_{n_{\mathrm{p}}, \ell_{\mathrm{u}}}^{\dagger}\right) .
\end{aligned}
$$

By defining $\mathbf{g}_{n_{\mathrm{p}}, \ell_{\mathrm{u}}} \triangleq \operatorname{vec}\left(\Delta_{n_{\mathrm{p}}, \ell_{\mathrm{u}}}^{\dagger}\right)$ and utilizing following equalities of the trace functions $\operatorname{Tr}\left(\mathbf{M}^{\dagger} \mathbf{Y} \mathbf{M}\right)=\operatorname{vec}(\mathbf{M})^{\dagger}\left(\mathbf{I}_{b} \otimes \mathbf{Y}\right) \operatorname{vec}(\mathbf{M})$, and $\operatorname{Tr}\left(\mathbf{T}^{\dagger} \mathbf{M}\right)=$ $\operatorname{vec}(\mathbf{T})^{\dagger} \operatorname{vec}(\mathbf{M})$ [8] where $\mathbf{Y}$ is the $a \times a$ matrix and $\mathbf{T}$ and $\mathbf{M}$ are the $a \times b$ matrices, (21) can be rewritten as

$$
\begin{aligned}
\beta_{n_{\mathrm{p}}, \ell_{\mathrm{u}}}= & \operatorname{Tr}\left(\hat{\mathbf{K}}_{n_{\mathrm{p}}, \ell_{\mathrm{u}}} \mathbf{R}_{\ell_{\mathrm{u}}} \hat{\mathbf{K}}_{n_{\mathrm{p}}, \ell_{\mathrm{u}}}^{\dagger}\right) \\
& +2 \Re\left[\operatorname{vec}\left(\mathbf{R}_{\ell_{\mathrm{u}}}^{\dagger} \hat{\mathbf{K}}_{n_{\mathrm{p}}, \ell_{\mathrm{u}}}^{\dagger}\right)^{\dagger} \mathbf{g}_{n_{\mathrm{p}}, \ell_{\mathrm{u}}}\right] \\
& +\mathbf{g}_{n_{\mathrm{p}}, \ell_{\mathrm{u}}}^{\dagger}\left(\mathbf{I}_{N_{\ell_{\mathrm{u}}}} \otimes \mathbf{R}_{\ell_{\mathrm{u}}}\right) \mathbf{g}_{n_{\mathrm{p}}, \ell_{\mathrm{u}}}
\end{aligned}
$$

and the condition $\left\|\Delta_{n_{\mathrm{p}}, \ell_{\mathrm{u}}}\right\|_{F} \leq \varepsilon_{n_{\mathrm{p}}, \ell_{\mathrm{u}}}$ is now rewritten by

$$
\mathbf{g}_{n_{\mathrm{p}}, \ell_{\mathrm{u}}}^{\dagger} \mathbf{I}_{M_{\ell_{\mathrm{u}}} L_{n_{\mathrm{p}}}} \mathbf{g}_{n_{\mathrm{p}}, \ell_{\mathrm{u}}} \leq \varepsilon_{n_{\mathrm{p}}, \ell_{\mathrm{u}}}^{2} .
$$

To handle the robust interference constraints in (20c) and (20d), we adopt the following lemma [16, p. 655], [8].

Lemma 1. S-Procedure lemma [16, p. 655]

Consider Y, $\mathbf{M}$ are the $n \times n$ Hermitian matrices; $\mathbf{b}$ is the complex vector size $n \times 1 ; c$, e are two real numbers; and assume the interior condition holds, i.e. there exists an $\overline{\mathbf{y}}$ satisfying $\overline{\mathbf{y}}^{\dagger} \mathbf{M} \overline{\mathbf{y}}<e$. Then two following inequalities

$$
\mathbf{y}^{\dagger} \mathbf{Y} \mathbf{y}+2 \Re\left(\mathbf{b}^{\dagger} \mathbf{y}\right)+c \geq 0 \text {, and } \forall \mathbf{y}^{\dagger} \mathbf{M y} \leq e
$$

hold if and only if there exists $\theta \geq 0$ such that

$$
\left[\begin{array}{cc}
\theta \mathbf{M}+\mathbf{Y} & \mathbf{b} \\
\mathbf{b}^{+} & c-\theta e
\end{array}\right] \succcurlyeq 0
$$

In spirit of Lemma 1 and light of (22) and (23), constraints (20c) and (20d) can be recast as LMIs as in (26) and (27), respectively (at the top of next page).

\subsection{An Iterative Algorithm Based on Convex Optimization}

The non-convex robust SRM problem (12) can be iteratively solved by solving the following convex problem

$$
\max _{\left\{\mathbf{Q}_{\mathrm{D}}, \mathbf{R}_{\mathrm{U}}\right\} \succcurlyeq 0,\{\theta\},\{\gamma\}} \phi_{\mathrm{Tot}}\left(\mathbf{Q}_{\mathrm{D}}, \mathbf{R}_{\mathrm{U}}\right)
$$

$$
\text { s.t. }(12 b),(12 c),(20 a),(20 b),(26),(27) \text {, }
$$




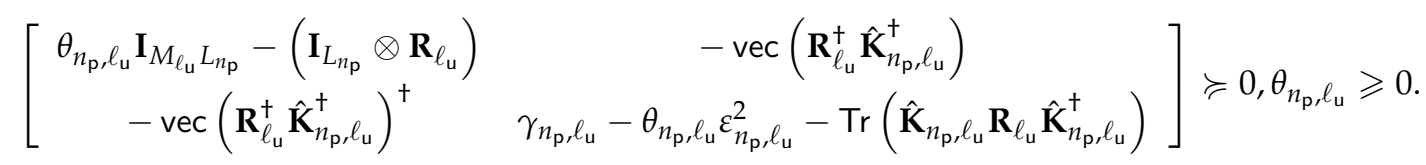

$$
\left[\begin{array}{cc}
\theta_{n_{\mathrm{p}}, \mathcal{B}} \mathbf{I}_{M_{\mathcal{B}} L_{n_{\mathrm{p}}}}-\left(\mathbf{I}_{L_{n_{\mathrm{p}}}} \otimes \mathbf{Q}_{k_{\mathrm{d}}}^{\text {sum }}\right) & -\operatorname{vec}\left(\left(\mathbf{Q}_{k_{\mathrm{d}}}^{\text {sum }}\right)^{\dagger} \hat{\mathbf{K}}_{n_{\mathrm{p}}, \mathcal{B}}^{\dagger}\right. \\
-\operatorname{vec}\left(\left(\mathbf{Q}_{k_{\mathrm{d}}}^{\text {sum }}\right)^{\dagger} \hat{\mathbf{K}}_{n_{\mathrm{p}}, \mathcal{B}}^{+}\right)^{\dagger} & \gamma_{n_{\mathrm{p}}, \mathcal{B}}-\theta_{n_{\mathrm{p}}, \mathcal{B}} \varepsilon_{n_{\mathrm{p}}, \mathcal{B}}^{2}-\operatorname{Tr}\left(\hat{\mathbf{K}}_{n_{\mathrm{p}}, \mathcal{B}} \mathbf{Q}_{k_{\mathrm{d}}}^{\text {sum }} \hat{\mathbf{K}}_{n_{\mathrm{p}}, \mathcal{B}}^{\dagger}\right.
\end{array}\right] \succcurlyeq 0, \theta_{n_{\mathrm{p}}, \mathcal{B}} \geqslant 0 .
$$

Table I: Simulation parameters

\begin{tabular}{l|l}
\hline Parameter & Value \\
\hline \hline Outer and inner cell radius & $40 \mathrm{~m}$ and $5 \mathrm{~m}$ \\
System bandwidth & $10 \mathrm{MHz}$ \\
Thermal noise power density & $-174 \mathrm{dBm} / \mathrm{Hz}$ \\
Noise figure & $\mathcal{B}: 13 \mathrm{~dB}, \mathrm{DLU}: 9 \mathrm{~dB}$ \\
Power budget at the BS, $P_{\mathcal{B}, \max }$ & $26 \mathrm{dBm}$ \\
Power budget at the ULUs, $P_{\mathrm{U}, \max }$ & $23 \mathrm{dBm}$ \\
Path loss between $\mathcal{B}$ and a user, $L_{\mathrm{BU}}$ & $103.8+20.9 \log _{10}(d) \mathrm{dB}$ \\
Path loss among users, $L_{\mathrm{UU}}$ & $145.4+37.5 \log _{10}(\hat{d}) \mathrm{dB}$ \\
SI cancellation capability [17], $C_{\mathrm{SI}}$ & $90 \mathrm{~dB}$ \\
\hline
\end{tabular}

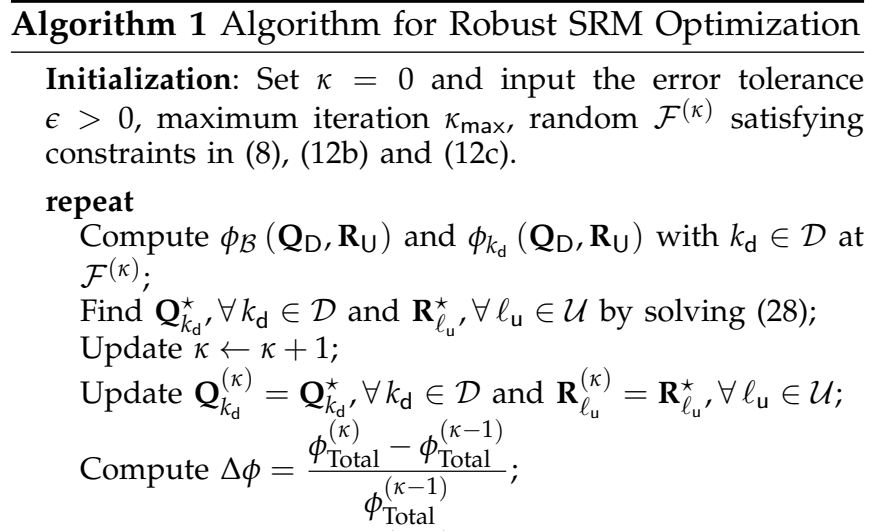

until tolerance reaches $|\Delta \phi| \leq \epsilon$ or number of iteration reaches $\kappa_{\max }$;

Output: $\mathbf{Q}_{k_{\mathrm{d}}}^{\mathrm{opt}}, \forall k_{\mathrm{d}} \in \mathcal{D}$ and $\mathbf{R}_{\ell_{\mathrm{u}}}^{\mathrm{opt}}, \forall \ell_{\mathrm{u}} \in \mathcal{U}$;

Find: $\mathbf{A}_{\ell_{\mathrm{u}}}^{\text {opt }}, \forall \ell_{\mathrm{u}} \in \mathcal{U}$ and $\mathbf{B}_{k_{\mathrm{d}}}^{\text {opt }}, \forall k_{\mathrm{d}} \in \mathcal{D}$ from (29).

where $\{\theta\} \triangleq\left\{\theta_{n_{\mathrm{p}}, \ell_{\mathrm{u}}}, \theta_{n_{\mathrm{p}}, \mathcal{B}}: \ell_{\mathbf{u}} \in \mathcal{U}, n_{\mathrm{p}} \in \mathcal{V}\right\}$, and $\{\gamma\} \triangleq$ $\left\{\gamma_{n_{\mathrm{p}}, \ell_{\mathrm{u}}}, \gamma_{n_{\mathrm{p}}, \mathcal{B}}: \ell_{\mathrm{u}} \in \mathcal{U}, n_{\mathrm{p}} \in \mathcal{V}\right\}$.

The problem (28) now can solved by utilizing convex optimization solver packages, such as CVX [18] or MOSEK [19]. The detailed procedure to handle the robust sum rate optimization problem is summarized in Algorithm 1. After obtaining the optimal solutions $\mathbf{Q}_{k_{\mathrm{d}}}^{\text {opt }}, \forall k_{\mathrm{d}} \in \mathcal{D}$ and $\mathbf{R}_{\ell_{u}}^{\mathrm{opt}}, \forall \ell_{\mathbf{u}} \in \mathcal{U}$ we compute the Cholesky decomposition $\mathbf{Q}_{k_{\mathrm{d}}}^{\mathrm{opt}}=\mathbf{M}_{k_{\mathrm{d}}}^{\mathrm{opt}}\left(\mathbf{M}_{k_{\mathrm{d}}}^{\mathrm{opt}}\right)^{\dagger}$ and $\mathbf{R}_{\ell_{\mathrm{u}}}^{\mathrm{opt}}=$ $\mathbf{T}_{\ell_{u}}^{\mathrm{opt}}\left(\mathbf{T}_{\ell_{\mathrm{u}}}^{\mathrm{opt}}\right)^{\dagger}$. Then, the optimal precoder matrices, i.e. $\mathbf{B}_{k_{\mathrm{d}}}^{\mathrm{opt}}$ and $\mathbf{A}_{\ell_{\mathrm{u}}}^{\mathrm{opt}}$, are given by

$$
\begin{aligned}
& \mathbf{B}_{k_{\mathrm{d}}}^{\mathrm{opt}}=\mathbf{M}_{k_{\mathrm{d}}}^{\mathrm{opt}}, \forall k_{\mathrm{d}} \in \mathcal{D}, \\
& \mathbf{A}_{\ell_{\mathrm{u}}}^{\mathrm{opt}}=\mathbf{T}_{\ell_{\mathrm{u}}^{\mathrm{opt}}}^{{ }_{\mathrm{u}}}, \forall \ell_{\mathbf{u}} \in \mathcal{U} .
\end{aligned}
$$

\section{Numerical Results}

In this section, the effectiveness of the robust sum rate maximization algorithm and impacts of imperfect CSI errors on system performance are validated through numerical simulations. The system parameters are set as: the number of ULU, DLU and PU are $U=D=P=$ 2 users; the each user is equipped with $M_{\ell_{\mathrm{u}}}=N_{k_{\mathrm{d}}}=2$ antennas for all $\ell_{\mathrm{u}}, k_{\mathrm{d}}$; BS is equipped $M_{\mathcal{B}}=N_{\mathcal{B}}=4$ antennas; the number of data-streams $d_{\ell_{\mathrm{u}}}=M_{\ell_{\mathrm{u}}}$, $d_{k_{\mathrm{d}}}=N_{k_{\mathrm{d}}}$. We also set $P_{\ell_{\mathrm{u}}, \max }=P_{\mathrm{U}, \max }, \forall \ell_{\mathrm{u}} \in \mathcal{U}$, and $\gamma_{n_{\mathrm{p}}}=\gamma_{\mathrm{PU}}, \forall n_{\mathrm{p}} \in \mathcal{V}$ with particular value in each simulation. Additionally, a single small cell model as in $[5,6]$ is adopted which includes a BS in the center and users uniformly and randomly distributed within the cell coverage. The uncertain NBE factors are set as a function of channel qualities, i.e. $\varepsilon_{n_{\mathrm{p}}, \mathcal{B}}=v\left\|\hat{\mathbf{K}}_{n_{\mathrm{p}}, \mathcal{B}}\right\|_{F}$ and $\varepsilon_{n_{\mathrm{p}}, \ell_{\mathrm{u}}}=v\left\|\hat{\mathbf{K}}_{n_{\mathrm{p}}, \ell_{\mathrm{u}}}\right\|_{F^{\prime}}$, with $v \in[0,1)$ [5]. The typical channel matrix gain is generated as $\mathbf{M}_{X}=\sqrt{\delta_{X}^{2}} \tilde{\mathbf{M}}$ with $\mathbf{M}_{X} \in\left\{\hat{\mathbf{K}}_{n_{\mathrm{p}}, \mathcal{B}}, \hat{\mathbf{K}}_{n_{\mathrm{p}}, \ell_{\mathrm{u}}}, \mathbf{F}_{k_{\mathrm{d}}, \ell_{\mathrm{u}}}, \mathbf{G}_{k_{\mathrm{d}}}, \mathbf{H}_{\ell_{\mathrm{u}}}\right\}$ and, the elements of the nominal small-scale fading $\tilde{\mathbf{M}}$ are randomly realized as independent complex Gaussian distribution with zero mean and unit variance. The values $\delta_{k_{d}}^{2} \delta_{\ell_{u}}^{2}$ $\delta_{n_{\mathrm{p}}, \mathcal{B}}^{2}$ follow the base station-to-user path loss $10^{-L_{\mathrm{BU}} / 10}$

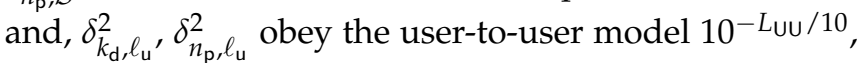
for all $k_{\mathrm{d}}, \ell_{\mathrm{u}}, n_{\mathrm{p}}$. The residual self-interference channel at BS follows $\mathbf{F}_{\mathcal{B}} \sim \mathcal{C N}\left(\sqrt{\frac{\delta_{\mathcal{B}}^{2} K}{1+K}} \tilde{\mathbf{F}}, \frac{\delta_{\mathcal{B}}^{2}}{1+K} \mathbf{I}_{M_{\mathcal{B}}} \otimes \mathbf{I}_{N_{\mathcal{B}}}\right)$ with $\delta_{\mathcal{B}}^{2}=10^{-C_{S I} / 10}$ and SI mitigation level $C_{S I}$, we also set Rician factor $K=1$ and deterministic matrix $\tilde{\mathbf{F}}$ to be the matrix of all ones $[13,14]$. Unless stated otherwise, the simulation parameters are given in Table I. The error tolerance of the iterative algorithm is set to $\epsilon=10^{-4}$ 


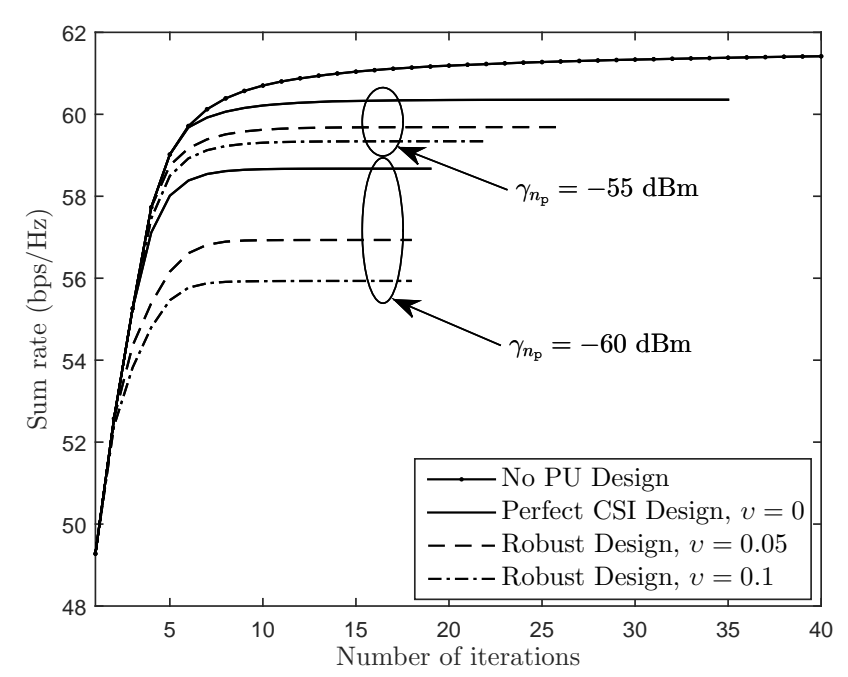

Figure 2: The convergence of the algorithm with various values of $\gamma_{\mathrm{PU}}$ and $v$ under a specific given channel and location realization.

and the maximum number of iterations is $\kappa_{\max }=100$. Furthermore, the elements in initial feasible set $\mathcal{F}^{(0)}$ is set as $\alpha \mathbf{I}$ with approriate dimensions and $\alpha$ is scaled to satisfy the constraints in (12).

Example 1: This simulation considers a specific random location and channel to investigate the convergence behaviour and the robustness of interference constraints. In Figure 2, the convergence of the iterative algorithm is depicted for difference values of uncertain channel errors $v$ and interference thresholds $\gamma_{\mathrm{PU}}$. As can be seen from this figure, the total sum rate first monotonically increases through about few tens of iterations, then converges at an optimal point. The sum rate performance of the CR network is degraded when the interference constraints are more restricted. It can be explained that the FD MU-MIMO CR network must trade-off its sum-rate performance for the guarantee of no harmful interference to the PUs. The FD cognitive users tend to reduce their transmit power when the allowable interference threshold is reduced. In addition, the system achievable sum-rate decreases for the larger value of imperfect CSI errors. This is due to that the users tend to reduce the transmit power to ensure the interference levels below an acceptable threshold even for the worst CSI errors.

In order to demonstrate the effectiveness of the robust algorithm in terms of guaranteeing interference constraints under the imperfect CSI, the cumulative distribution function (CDF) of interference power at PU is plotted in Figure 3. This figure is simulated for 100 random channels with 50 random channel uncertainties in each and fixed user locations. It can be seen that the robust design guarantees that the PU interference power is always smaller than the allowable threshold of $\gamma_{\mathrm{PU}}=-60 \mathrm{dBm}$ (i.e., $10^{-9} \mathrm{~W}$, highlighted by a vertical line in Figure 3). On the contrary, the non-robust design exhibits the violation of interference constraints about $40 \%$ of channel realizations.

Example 2: In this example, we investigate the system

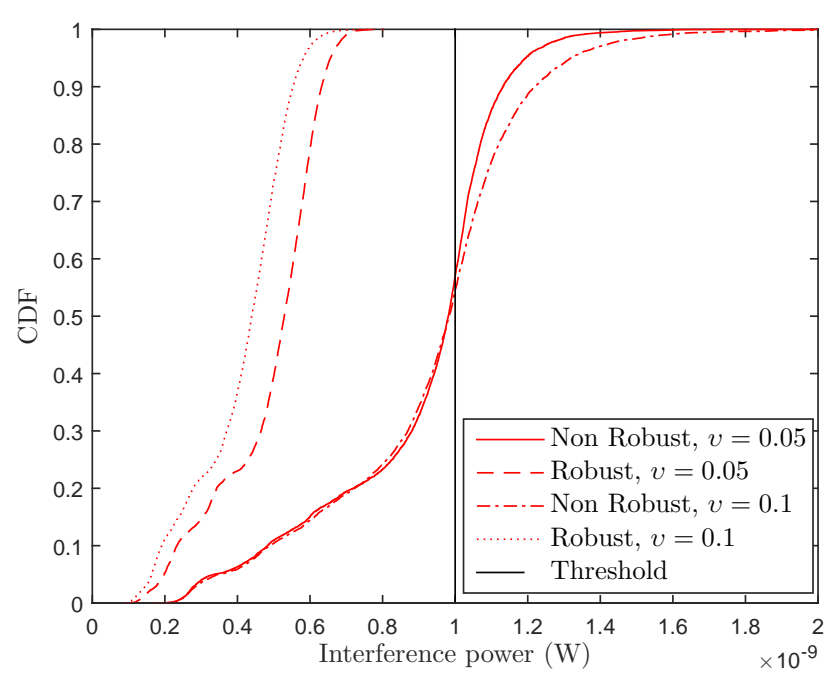

Figure 3: The CDF of interference power with difference values $v$.

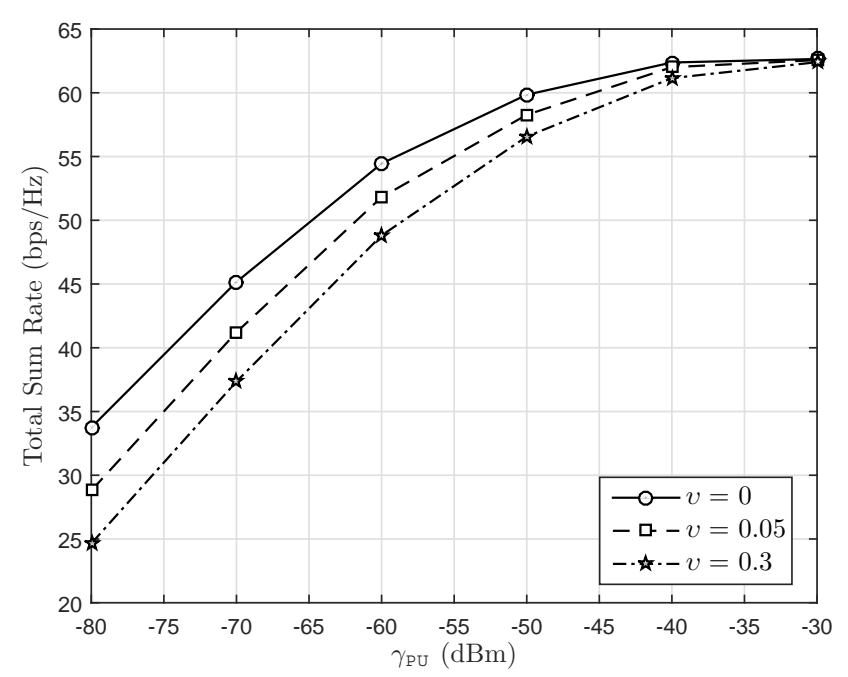

Figure 4: The average achievable sum-rate versus allowable interference thresholds for difference values of $v$.

performance by Monte Carlo simulations of 100 runs of user locations and channel realizations. Figure 4 demonstrates the average sum rate versus interference thresholds $\gamma_{\mathrm{PU}}$. It is clear that the average achievable sum rate increases as the allowable interference threshold increases. An increase in the imperfect CSI errors degrades the system sum rate. However, the achievable sum-rate is not significantly affected by the CSI errors when the interference threshold is large enough. The reason is that the SUs can transmit the maximum transmit power when the interference constraints are relaxed and almost all SU transmissions are likely to satisfy the interference constraints for all possible CSI errors.

\section{Conclusion}

This paper has presented an optimization algorithm which robustly designs precoding matrices for a secondary FD MU-MIMO network under imperfect CSI of SU-PU links. The problem of interest is to maximize 
the sum-rate of the secondary network under the SUs and BS transmit power constraints and PU interference power constraints. To deal with the non-concavity of the sum-rate objective function and the semi-infinite robust interference constraints, we have adopted d.c. program and the LMIs of S-Procedure to transform the design problems into a successive convex program. The numerical simulation results have demonstrated the designed precoders are robust to the CSI imperfectness. The results have also revealed the impacts of the interference thresholds and the CSI errors on the sum-rate performance of the CR networks.

\section{ACKNOWLEDGMENT}

This research is funded by Vietnam National Foundation for Science and Technology Development (NAFOSTED) under grant number 102.04-2017.308.

\section{REFERENCES}

[1] D. Nguyen, L.-N. Tran, P. Pirinen, and M. Latva-aho, "Precoding for full duplex multiuser MIMO systems: Spectral and energy efficiency maximization," IEEE Transactions on Signal Processing, vol. 61, no. 16, pp. 40384050, 2013.

[2] S. Huberman and T. Le-Ngoc, "Full-duplex MIMO precoding for sum-rate maximization with sequential convex programming," IEEE Transactions on Vehicular Technology, vol. 64, no. 11, pp. 5103-5112, 2015.

[3] A. C. Cirik, R. Wang, Y. Hua, and M. Latva-aho, "Weighted sum-rate maximization for full-duplex MIMO interference channels," IEEE Transactions on Communications, vol. 63, no. 3, pp. 801-815, 2015.

[4] H. H. M. Tam, H. D. Tuan, and D. T. Ngo, "Successive convex quadratic programming for quality-of-service management in full-duplex MU-MIMO multicell networks," IEEE Transactions on Communications, vol. 64, no. 6, pp. 2340-2353, June 2016.

[5] A. C. Cirik, S. Biswas, S. Vuppala, and T. Ratnarajah, "Robust transceiver design for full duplex multiuser MIMO systems," IEEE Wireless Communications Letters, vol. 5, no. 3, pp. 260-263, 2016.

[6] P. Aquilina, A. C. Cirik, and T. Ratnarajah, "Weighted sum rate maximization in full-duplex multi-user multicell MIMO networks," IEEE Transactions on Communications, vol. 65, no. 4, pp. 1590-1608, 2017.

[7] E. A. Gharavol, Y. Liang, and K. Mouthaan, "Robust linear transceiver design in MIMO ad hoc cognitive radio networks with imperfect channel state information," IEEE Transactions on Wireless Communications, vol. 10, no. 5, pp. 1448-1457, 2011.

[8] Y. Zhang, E. DallAnese, and G. B. Giannakis, "Distributed optimal beamformers for cognitive radios robust to channel uncertainties," IEEE Transactions on Signal Processing, vol. 60, no. 12, pp. 6495-6508, 2012.

[9] H. H. Kha, T. T. Vu, and T. Do-Hong, "Energy-efficient transceiver designs for multiuser MIMO cognitive radio networks via interference alignment," Telecommunication Systems, vol. 66, no. 3, pp. 469-480, Nov 2017.

[10] H. H. Kha and H. Q. Ta, "Min-max MSE-based interference alignment for transceiver designs in cognitive radio networks," Radioengineering, vol. 26, no. 1, pp. 170178, 2017.
[11] V.-D. Nguyen, L.-N. Tran, T. Q. Duong, O. Shin, and R. Farrell, "An efficient precoder design for multiuser MIMO cognitive radio networks with interference constraints," IEEE Transactions on Vehicular Technology, vol. 66, no. 5, pp. 3991-4004, 2017.

[12] V.-D. Nguyen, C. T. Nguyen, H. V. Nguyen, and O. Shin, "Joint beamforming and antenna selection for sum rate maximization in cognitive radio networks," IEEE Communications Letters, vol. 21, no. 6, pp. 1369-1372, 2017.

[13] A. C. Cirik, R. Wang, Y. Rong, and Y. Hua, "MSE-based transceiver designs for full-duplex MIMO cognitive radios," IEEE Transactions on Communications, vol. 63, no. 6, pp. 2056-2070, 2015.

[14] A. C. Cirik, S. Biswas, O. Taghizadeh, and T. Ratnarajah, "Robust transceiver design in full-duplex MIMO cognitive radios," IEEE Transactions on Vehicular Technology, vol. 67, no. 2, pp. 1313-1330, 2018.

[15] H. H. Kha, H. D. Tuan, and H. H. Nguyen, "Fast global optimal power allocation in wireless networks by local D.C. programming," IEEE Transactions on Wireless Communications, vol. 11, no. 2, pp. 510-515, 2012.

[16] S. Boyd and L. Vandenberghe, Convex optimization. Cambridge university press, 2004.

[17] D. Bharadia and S. Katti, "Full duplex MIMO radios," in Proceedings of the 11th USENIX Conference on Networked Systems Design and Implementation. Berkeley, CA, USA: USENIX Association, 2014, pp. 359-372.

[18] M. Grant, S. Boyd, and Y. Ye, "Cvx: Matlab software for disciplined convex programming," 2008.

[19] A. Mosek, "The mosek optimization toolbox for matlab manual," 2015.

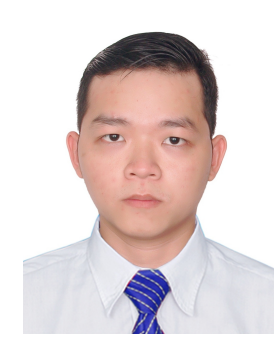

Xuan-Xinh Nguyen received the B.S. degree in Electronics and Telecommunications Engineering from Ho Chi Minh City University of Technology and Education, Vietnam, in 2015. He is currently pursuing the M.Eng. degree in Telecommunications Engineering at the Faculty of Electrical and Electronics Engineering, Ho Chi Minh City University of Technology. His research interests include full-duplex transmission, cognitive radio, and MIMO communications.

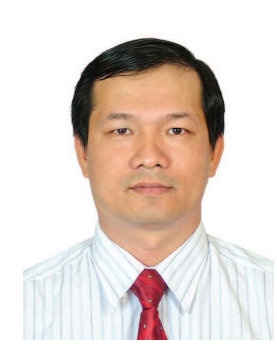

Ha Hoang Kha received the B.Eng. and M.Eng. degrees from Ho Chi Minh City University of Technology, in 2000 and 2003, respectively, and the Ph.D. degree from the University of New South Wales, Sydney, Australia, in 2009, all in Electrical Engineering and Telecommunications. From 2000 to 2004, he was a research and teaching assistant with the Department of Electrical and Electronics Engineering, Ho Chi Minh City University of Technology. He was a visiting research fellow at the School of Electrical Engineering and Telecommunications, the University of New South Wales, Australia, from 2009 to 2011. He was a postdoctoral research fellow at the Faculty of Engineering and Information Technology, University of Technology Sydney, Australia from 2011 to 2013. He is currently a lecturer at the Faculty of Electrical and Electronics Engineering, Ho Chi Minh City University of Technology, Vietnam. His research interests are in digital signal processing and wireless communications, with a recent emphasis on convex optimization techniques in signal processing for wireless communications. 